\title{
Redundancy gains and coactivation with two different targets: The problem of target preferences and the effects of display frequency
}

\author{
J. TOBY MORDKOFF and JEFF MILLER \\ University of California, San Diego, La Jolla, California
}

\begin{abstract}
When a visual display contains two targets, both of which require the same response, reaction times (RTs) are faster than when only one target appears. This effect has previously been obtained regardless of whether the redundant targets are the same or different in shape, and in at least one set of two-target experiments, the redundancy gains have been larger for different targets (Grice \& Reed, 1992). Experiments with two different targets have also revealed violations of the race-model inequality, suggesting that redundant targets coactivate the response (Miller, 1982). The present paper reexamines both of these findings, because both appear to be inconsistent with the interactive race model (Mordkoff \& Yantis, 1991). Experiment 1 shows that the race-model inequality is not violated when the experimental design is free of biased contingencies; Experiment 1 also provides evidence that target preferences may artifactually produce the RT advantage for different- over same-target trials. Experiment 2, however, shows that the race-model inequality is violated when the frequencies of single- and redundant-target displays are equated (without introducing any biased contingencies), implying that the interactive race model cannot account for the results of experiments involving more than one type of target. Alternative loci for coactivation are briefly discussed.
\end{abstract}

When people are given more than one reason to perform some behavioral act, they often perform it more quickly (or are simply more likely to do it) than when they are given only one reason. In the real world, reasons are usually given sequentially, and it is easy to imagine that their influence arises from serial processing. In the laboratory, however, the same effects can be observed when all of the reasons are given at once, and the data suggest that people can divide their attention and process multiple inputs in parallel. For example, when subjects are required to press a button (i.e., make a go response) if presented with one or more targets, and to do nothing (no-go) if no targets appear, responses have been shown to be faster (see, e.g., Grice, Canham, \& Boroughs, 1984; Miller, 1982; Mordkoff \& Yantis, 1991; van der Heijden, Schreuder, Maris, \& Neerincx, 1984) and more likely (see, e.g., Duncan, 1980; Shaw, 1982) when more than one target appears in the display. This advantage for trials with more than one target is usually called a redundancy gain, because any additional targets are redundant with the first.

Theorists have proposed two different classes of parallelprocessing model that can account for redundancy gains:

This work was supported by Public Health Service Training Grant T32-MH14268 and Grant R01-MH40733 from the National Institutes of Mental Health. We thank Charles Eriksen, Patricia Haden, Cathleen Moore, J. T. Townsend, Rolf Ulrich, and two anonymous reviewers for advice and comments, and Dana Feinstein and Rima Kliore for help in conducting the experiments. Correspondence should be sent to J. T. Mordkoff at the Department of Psychology, Bryn Mawr College, Bryn Mawr, PA 19010 (e-mail: mordkoff@cc.brynmawr.edu). separate activation (or race models), and coactivation. ${ }^{1}$ Separate-activation models posit that redundant targets are processed separately and in parallel, with each of the targets racing to activate the response. These models explain redundancy gains in terms of statistical facilitation (Raab, 1962): If the time required for a target to trigger a response varies stochastically across trials, the average finishing time for the faster of two parallel processes will be shorter than the average finishing time for either process alone.

In contrast, coactivation models posit that the information supporting a response is somehow pooled across processing channels prior to response execution. Thus, rather than racing to trigger a response, redundant targets are said to coactivate the response (Miller, 1982). Clearly, the response will be triggered more quickly with two sources of information contributing activation than with only one, so this class of models can also explain the reaction time (RT) advantage for redundant-target trials.

Because both separate-activation and coactivation models can explain redundancy gains, mean RT cannot be used to discriminate between these two classes of models. However, Miller $(1978,1982)$ has shown that models assuming separate activations must obey the following rule-known as the race-model inequality-that coactivation models need not obey. The inequality concerns the entire distribution of RTs to single- and redundant-target trials:

$$
P\left(\mathrm{RT}<t \mid \mathrm{T}^{1} \& \mathrm{~T}^{2}\right) \leq P\left(\mathrm{RT}<t \mid \mathrm{T}^{1}\right)+P\left(\mathrm{RT}<t \mid \mathrm{T}^{2}\right),
$$


where $t$ is the time since display onset and $\mathrm{T}^{L}$ denotes that a target was presented in location $L$. In effect, the racemodel inequality places an upper bound on the speed of redundant-target responses throughout the entire distribution of observed RTs (for further elaboration, see Colonius, 1990; Diederich, 1992; Townsend \& Nozawa, 1992; Ulrich \& Giray, 1986). Early tests of separateactivation models using the race-model inequality (e.g., Grice et al., 1984; Miller, 1982; van der Heijden et al., 1984) produced somewhat equivocal results. Some experiments revealed violations of the inequality, a result that rules out the separate-activation model; in other experiments, the inequality was satisfied.

Mordkoff and Yantis (1991) noted that violations of the race-model inequality have only been observed when biased interstimulus or nontarget-response contingencies were included within the experimental design (cf. the notion of internal constraint; Garner, 1962). A biased interstimulus contingency exists when the presence of a certain distractor predicts the absence of a certain target (relative to the prediction made by another target); a biased nontarget-response contingency exists when the presence of a distractor predicts that no response should be made (relative to the baseline probability; see Mordkoff \& Yantis, 1991, for quantitative details). For example, if the overall probability that a response should be made is .5 , but the probability that a response should be made given that the nontarget letter $I$ is present is only .25 , then the design includes a nontarget-response contingency bias against single-target trials that involve the nontarget $I$. An analysis of the contingencies within the designs of previously reported redundant-target experiments, as well as the results from several new experiments in which the two types of contingency were systematically manipulated, suggested that biased contingencies are a necessary condition for violations of the race-model inequality to be observed.

These observations led Mordkoff and Yantis (1991) to propose the interactive race model. The model retains the central idea from the separate-activation model-that of a race between target signals-but discards the assumption that the channels are independent. According to the model, each target represents a separate opportunity for a go response to be triggered, but mechanisms sensitive to contingencies within the experimental design may also provide simultaneous input to either stimulus identification or response selection. When no biased contingencies are present within the experimental design, the interactive race model predicts that the race-model inequality will always be satisfied; the model also predicts no violations when contingencies are biased against redundant-target trials. These predictions have been confirmed by several experiments (e.g., Miller, 1982, Experiment 5; Mordkoff \& Yantis, 1991, Experiments 1 and 4; van der Heijden et al., 1984). The model also allows for violations of the race-model inequality when the experimental design contains contingencies that are biased to favor redundanttarget trials, and these have also been observed in empirical work (e.g., Miller, 1982, Experiments 4 and 5; Mordkoff \& Yantis, 1991, Experiments 2, 3, and 5).

In summary: when subjects are required to make a go response when presented with one or more of a specific visual target, and to withhold their response when no targets appear, the data indicate that redundant targets are processed in parallel. Most of the data are also consistent with the view that each target instance is processed independently - as shown by satisfaction of the race-model inequality and other tests (see Mordkoff \& Yantis, 1991)-but this holds only when the experimental design includes no biased contingencies. If biased contingencies are introduced, then target identification and/or response activation appear to become interdependent, and violations of the race-model inequality may be observed.

Overview. Most of the previous research concerning redundancy gains has examined performance on relatively simple, visual tasks. In particular, most studies have involved only one type of target (e.g., the letter $X$ ). An open question, therefore, is whether the interactive race model also applies to more complex tasks involving more than one type of target. The present experiments were designed to address this question. The specific question we ask is whether-in multitarget tasks-violations of the racemodel inequality are only found when contingencies are biased in favor of redundant-target trials.

\section{EXPERIMENT 1}

Grice and Reed (1992) have recently reported a pair of target-detection experiments involving more than one type of target; thus, their results are quite relevant to the present concerns. In their first experiment, the two different targets-both mapped onto the same go responsewere the upper- and lowercase forms of a single letter (e.g., $A$ and $a$ ). In their second experiment, the two targets were completely different letters (e.g., $A$ and $D$ ). The design used for both experiments included a contingency bias in favor of redundant-target trials. In particular, by Equations 4 and 6 of Mordkoff and Yantis (1991, pp. 523524), there was an interstimulus-contingency benefit of +.083 , while the nontarget-response contingency benefit was zero.

The use of two different types of target allowed Grice and Reed (1992) to perform two tests of the race-model inequality: one using the redundant-target RTs from trials with two identical targets (hereafter, the same-target condition), and another using the RTs from trials with two different targets (the different-target condition). Significant violations of the race-model inequality were observed in both comparisons. These results are consistent with the interactive race model, because the experimental design included a bias in favor of redundant-target trials. The finding of violations in the test involving the differenttarget condition is particularly interesting, because it suggests that targets can interact (via interchannel crosstalk) even when they have different names and/or shapes. However, it must be noted that these data do not provide strong 
evidence in favor of the interactive race model, because violations of the race-model inequality are also consistent with coactivation. In order to conclude more firmly in favor of the interactive race model, it is necessary to show that violations disappear when the design is free of biased contingencies. Our primary purpose in Experiment 1 was to conduct this crucial test; therefore, we used an unbiased design.

A secondary purpose of Experiment 1 was to reexamine an aspect of Grice and Reed's (1992) data that is difficult to reconcile with the interactive race model: the consistent RT advantage for redundant-target displays with two different targets over displays with two identical targets (hereafter, the different-target advantage). This finding contradicts all current models of divided attention. If redundant targets are processed separately (as under race models), it should not matter whether the two targets are the same or different. Similarly, according to coactivation models, all evidence concerning the presence of at least one target is pooled, and it would seem likely that the activations from identical targets could be combined at least as easily as those from different targets (Fournier \& Eriksen, 1990; Miller, 1991). Finally, the differenttarget advantage cannot be explained by the interactive race model, because the contingencies were equal for the same- and different-target conditions in the experiments done by Grice and Reed.

If the different-target advantage truly reflects faster processing of different targets than of same targets, then it will require some explanation before the interactive race model can be generalized to two-target tasks. However, such an explanation may not be needed, because the advantage could arise as an artifact of target preferences within certain types of spatially parallel processing models. Assume for the moment that each of the subjects in a two-target experiment has a preference for one of the two targets, such that it can be identified as a target more rapidly than the other, nonpreferred target. On different-target trials there will always be one preferred target (as well as one nonpreferred target); in contrast, half of the same-target trials will only involve the nonpreferred target. Thus, half of the same-target RTs should be slower than the different-target RTs. This could cause an artifactual different-target advantage-one not due to any real advantage for trials with different targets, but due instead to target preferences.

This may be demonstrated more formally for the class of models in which processing is a spatially parallel and self-terminating race, so that response time on a redundanttarget trial is the minimum of the times needed to detect each of the two targets (plus an independent, additive component reflecting extraneous perceptual and motor latencies, which we shall here ignore). Let $T_{1}$ and $T_{2}$ be random variables representing the times needed to detect Targets 1 and 2 within a given channel, respectively (we shall here omit location superscripts and assume no favored positions-that is, $T_{1}$ has the same distribution in the upper and lower channels). On different-target trials, response time is

$$
\mathrm{RT}_{\mathrm{DT}}=\min \left(T_{1}, T_{2}\right),
$$

and on same-target trials, response time is

$$
\mathrm{RT}_{\mathrm{ST}}=\left\{\begin{array}{l}
\min \left(T_{1}, T_{1}\right), \text { with } P=.5, \\
\min \left(T_{2}, T_{2}\right), \text { with } P=.5
\end{array}\right.
$$

(assuming equal numbers of same-target trials with each of the two possible targets). If the two target-detection latencies on a given redundant-target trial are stochastically independent, then

$$
P\left(\mathrm{RT}_{\mathrm{DT}}<t\right)=1-\left[P\left(T_{1} \geq t\right) \times P\left(T_{2} \geq t\right)\right]
$$

and

$$
P\left(\mathrm{RT}_{\mathrm{ST}}<t\right)=\frac{\left[1-P\left(T_{1} \geq t\right)^{2}\right]+\left[1-P\left(T_{2} \geq t\right)^{2}\right]}{2} .
$$

Therefore,

$$
\begin{aligned}
& P\left(\mathrm{RT}_{\mathrm{DT}}<t\right)-P\left(\mathrm{RT}_{\mathrm{ST}}<t\right) \\
& \quad=\frac{\left[P\left(T_{1} \geq t\right)-P\left(T_{2} \geq t\right)\right]^{2}}{2} \geq 0 .
\end{aligned}
$$

Thus, as long as the two targets are detected at different rates [i.e., $P\left(T_{1}<t\right) \neq P\left(T_{2}<t\right)$ for some $t$ ], the cumulative distribution function for $\mathrm{RT}_{\mathrm{DT}}$ will dominate that for $\mathrm{RT}_{\mathrm{ST}}$, causing a different-target advantage in mean $\mathrm{RT}$. Clearly one scenario that will produce this effect is when subjects prefer one target over another [e.g., $P\left(T_{1}<t\right) \geq$ $P\left(T_{2}<t\right)$ for all $\left.t\right]^{2}$

To counteract the possible effects of target preferences, we used a method of analysis that was originally developed to counteract the analogous effects of positional or dimensional preferences (see Biederman \& Checkosky, 1970; Miller \& Lopes, 1988; Mullin, Egeth, \& Mordkoff, 1988). Specifically, we checked each subject's same-target data for evidence of a target preference (details below). This was done for each subject separately. If no evidence of a preference was found, we used the mean of all sametarget RTs as that subject's same-target mean RT (as did Grice \& Reed, 1992). If evidence of a preference was found, however, we used the average of the RTs from the same-target trials involving two preferred targets as that subject's same-target mean RT. If the different-target advantage in the overall means represents some real benefit of having two different targets within the displayone that extends beyond target preferences-we should still observe an advantage in using this conservative comparison. In contrast, if the different-target advantage is due to the existence of target preferences, it should not be present when the analysis corrects for this artifact.

\section{Method}

Subjects. Twenty undergraduate students at the University of California, San Diego, participated to partially fulfill an introductory course requirement. All subjects reported normal or correctedto-normal visual acuity.

Apparatus and Stimuli. The stimuli were presented on NEC Multisync color monitors controlled by VGA graphics adapters. The subjects responded by pressing a button on a custom response box with the dominant index finger. 
Each display included two white letters on a black background, one above and one below fixation. Each subject was given a different mapping of five randomly selected consonants as Target 1 , Target 2, the distractor, and the two noise letters. (We here define a distractor as a nontarget that may appear in the same display as a target, and a noise letter as a nontarget that never appears with a target.) From a viewing distance of $45 \mathrm{~cm}$, each letter subtended $1.40^{\circ} \times 0.89^{\circ}$ visual angle and the display locations were each $1.53^{\circ}$ from fixation. The fixation cross was $0.64^{\circ} \times 0.64^{\circ}$.

Design. Each block included approximately 55 trials: 48 testing trials, plus 5 random warm-up trials and a random recovery trial after each error. Half of all trials included at least one target and required a response; the other half included no targets and required that the subject not respond. In each block of 48 testing trials, 16 trials included only one target (4 with each target in each location), 4 included both targets, and 4 included two identical targets; the remaining 24 trials included neither target (of which exactly 4 included two identical letters). The entire design, in terms of trial frequencies, is given in Table 1.

The design included no biased contingencies: The presence of a target in one location provided the same information concerning the identity of the letter in the opposite location as did the presence of the distractor. Also, the probability that a response was required, given the presence of the distractor, was the same as the overall probability of a response. Finally, the probability that a response should be made was the same for trials with identical and nonidentical letters.

Procedure. Each subject participated in a single session lasting about $50 \mathrm{~min}$. After a short (20-trial) practice block during which the subjects were given RT and accuracy feedback on each trial, there were 12 blocks of testing trials. Unknown by the subjects, the first 2 full blocks were also considered practice. Data from all warm-up and recovery trials (i.e., the first five trials in a block and the trials immediately following each error) were also excluded from the analysis.

Each trial began with the presentation of a fixation cross at the center of the screen for $750 \mathrm{msec}$. After a 100 -msec blank interval, the stimulus appeared. As soon as a response was made, whether correct or a false alarm, the stimulus was removed. If no response had been made by $1,000 \mathrm{msec}$, the test display was removed and the trial was considered a no-go or a "miss" error. The intertrial interval was $2,200 \mathrm{msec}$. A $700-\mathrm{Hz}$ tone sounded for $200 \mathrm{msec}$ following all false-alarm and miss errors. After the short series of practice trials, RT feedback was only displayed during an enforced 7 -sec break at the end of a block.

Data analysis. Three separate analyses were initially conducted. The first concerned redundancy gains in overall mean RT. This analysis tested whether responses to redundant-target displays were significantly faster than those to single-target displays by comparing (within subjects) the mean RT on redundant-target trials to the mean RT on single-target trials. Separate analyses were conducted for the same- and different-target conditions.

The second analysis also examined redundancy gains, but used the modified form of the conservative test (Biederman \& Checkosky, 1970), as suggested by Miller and Lopes (1988). This test corrects for positional preferences. Both redundancy gain analyses were preceded by an analysis of variance (ANOVA) involving practice (two levels: Blocks 3-7, and Blocks 8-12) and condition (three levels: single target, same targets, and different targets); if a significant interaction was observed, separate tests for redundancy gains were conducted using the data from Blocks 3-7 and Blocks 8-12. ${ }^{3}$

The third analysis checked for violations of the race-model inequality (for details on the method, see Mordkoff \& Yantis, 1991). Again, separate tests were conducted for the same- and differenttarget conditions. The data were first corrected for fast guesses by using the "twin-killing" method (Eriksen, 1988; Miller \& Lopes, 1991). For all of these tests, the cumulative distribution functions (CDFs) were evaluated at the 5 th- 95 th percentiles at $5 \%$ intervals. To reduce the influence of practice effects on the shape of the final CDFs, separate CDFs were made using the data from Blocks 3-7 and Blocks 8-12, and separate tests of the race-model inequality were conducted. To produce graphical representations, the mean values of RT were calculated across subjects at each quantile (i.e., the CDFs were Vincentized).

\section{Results}

Initial analyses. The mean RTs from Experiment 1 appear in Table 2. The ANOVA examining practice (Blocks 3-7 vs. 8-12) and target condition (single, same, different) revealed a significant main effect of practice $[F(1,19)=4.42, p<.05]$ and a significant interaction between practice and target condition $[F(2,38)=5.05$, $p<.025]$. Therefore, separate redundancy-gain tests were conducted for Blocks 3-7 and Blocks 8-12. Significant redundancy gains were observed in all comparisons using the overall means [Blocks 3-7, $t(19)=6.67$ and 8.42 for same and different targets, respectively; Blocks $8-12, t(19)=4.01$ and 11.88 for same and different targets, respectively; all $p s<.001]$. Nine of the 20 subjects showed a positional preference (at $p<.10$; see Miller \& Lopes, 1988). Correcting for this lowered the estimates of the single-target means (see Table 2), but the redundancy gains were all still significant (all $p$ s $<.005$ or better). In the redundant-target RTs, we observed an insignificant different-target advantage during Blocks 3-7 $[t(19)=1.10, p>.10]$. However, during Blocks 8-12, different-target trials were responded to about $11 \mathrm{msec}$ faster than same-target trials $[t(19)=2.88, p<.005]$.

The tests using the race-model inequality revealed no violations (see Figure 1). At no quantile was the sum of the single-target CDFs below the CDF for the same- or different-target condition; this was true for Blocks 3-7 and Blocks 8-12.

Analysis of target preferences. In order to determine whether a given subject exhibited a target preference, we

Table 1

Display Frequencies (Per Block of 48 Trials), Experiment 1

\begin{tabular}{cccccc}
\hline & \multicolumn{5}{c}{ Upper Location } \\
\cline { 2 - 6 } Lower Location & Target 1 & Target 2 & Distractor & Noise 1 & Noise 2 \\
\hline Target 1 & 2 & 2 & 4 & 0 & 0 \\
Target 2 & 2 & 2 & 4 & 0 & 0 \\
Distractor & 4 & 4 & 0 & 4 & 4 \\
Noise 1 & 0 & 0 & 4 & 2 & 2 \\
Noise 2 & 0 & 0 & 4 & 2 & 2 \\
\hline
\end{tabular}


Table 2

Mean Reaction Times (in Milliseconds), Experiment 1 Condition

Blocks 3-7 Blocks 8-12

Single target, overall

Single target, conservative*

Same targets, overall

Same targets, conservative $\dagger$

Different targets

*Corrected for favored positions. +Corrected for preferred targets.

performed a $t$ test comparing the RTs from all trials with one or two of the first target against all trials with one or two of the second target. If a subject showed a preference (at $p<.10$ ), the faster same-target mean was retained; if the subject did not exhibit a preference, the mean of all same-target trials was used for that subject.

Twelve of the 20 subjects exhibited a target preference; across all 20 subjects, the mean difference between same preferred targets (i.e., same-target trials with two of the preferred target) and same nonpreferred targets was 15 msec for both Blocks 3-7 $[t(19)=3.15, p<.005]$ and Blocks $8-12[t(19)=2.57, p<.01]$. Similar advantages for the preferred target were also observed on

\section{Experiment 1}
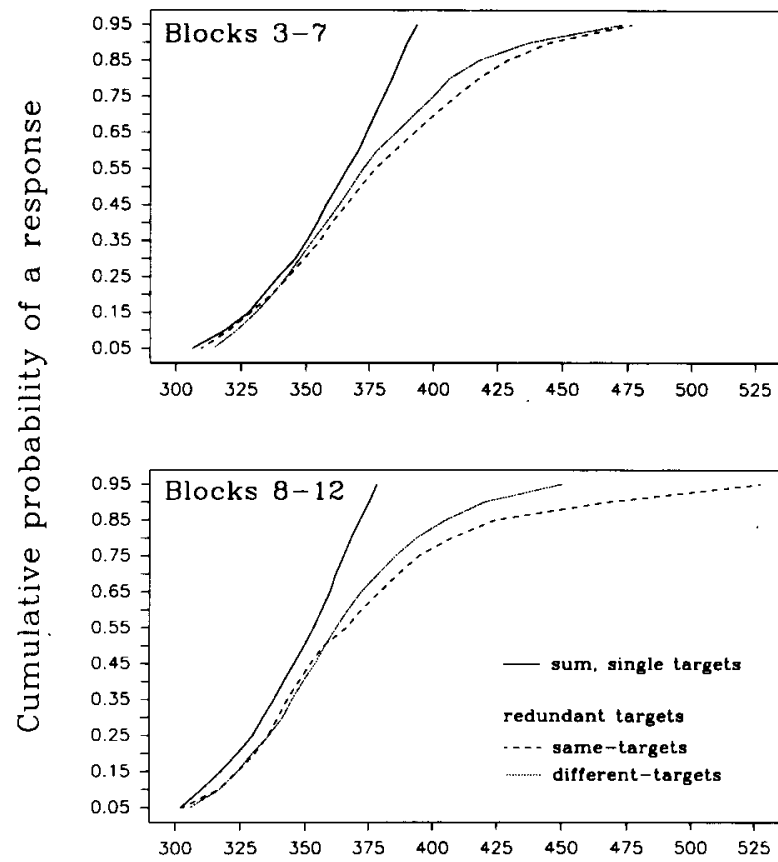

Time since stimulus onset (ms)

Figure 1. Cumulative distribution functions (CDFs) for Blocks 3-7 (upper panel) and Blocks 8-12 (lower panel), Experiment 1. (In each panel, the solid line represents the sum of the two single-target CDFs. The broken lines represent the redundant-target CDFs, with a dashed line for the same-target condition, and a dotted line for the differenttarget condition. There are no violations of the race-model inequality in any comparison.) the single-target trials (see Table 2). Most importantly, for Blocks 8-12 (wherein a different-target advantage was observed in the overall means), the mean RT for same preferred targets was nearly the same as that for different targets $[t(19)=0.73]$. Thus, there is no evidence of a different-target advantage when target preferences are taken into account.

Accuracy. Overall, the subjects were very accurate, making fewer than $0.2 \%$ miss errors and $1.8 \%$ false alarms. The miss errors were equally distributed among the various single- and redundant-target trials.

\section{Discussion}

The race-model inequality was everywhere satisfied in this experiment. This result was predicted by the interactive race model, because the design included no biased contingencies. These data contrast with those of Grice and Reed (1992); in their experiments, which had a design that included a contingency bias favoring redundant-target trials, significant violations of the race-model inequality were observed. Taken together, therefore, these experiments support the view that biased contingencies are necessary for violations of the race-model inequality. This pattern of results supports the interactive race model.

The present experiment also revealed a significant different-target advantage (i.e., faster responses for different-target trials when compared with all same-target trials), albeit only during Blocks 8-12. However, at least in our data, this advantage appears to be an artifact of target preferences: RTs in the different-target condition were not significantly faster than those in the samepreferred-target condition. From this we infer that no special interpretive weight should rest on the different-target advantage. Because over half of our subjects showed a preference for one target over the other, it is plausible that target preferences also had an effect on the data presented by Grice and Reed (1992). ${ }^{4}$

Summarizing our findings to this point: the results from experiments involving two types of target appear to be very similar to those from one-target tasks. Both sets of data are consistent with the interactive race model, in that violations of the race-model inequality have only been observed under experimental designs that include biased contingencies (e.g., Grice \& Reed, 1992; Mordkoff \& Yantis, 1991, Experiments 2, 3, and 5), whereas the race-model inequality has always been satisfied under designs that are free of biased contingencies (e.g., the present Experiment 1; Mordkoff \& Yantis, 1991, Experiments 1 and 4). The one difference is that the data from two-target experiments may be affected by target-preference artifacts, something that is impossible when there is only one type of target.

\section{EXPERIMENT 2}

Before concluding that the interactive race model applies to two-target tasks, as it does to one-target tasks, it is necessary to consider the effects of display frequency. 
Table 3

Display Frequencies (Per Block of 48 Trials), Experiment 2

\begin{tabular}{lccccccc}
\hline & \multicolumn{5}{c}{ Upper Location } \\
\cline { 2 - 7 } Lower Location & Target 1 & Target 2 & Distractor 1 & Distractor 2 & Noise 1 & Noise 2 \\
\hline Target 1 & 2 & 2 & 2 & 2 & 0 & 0 \\
Target 2 & 2 & 2 & 2 & 2 & 0 & 0 \\
Distractor 1 & 2 & 2 & 0 & 0 & 2 & 2 \\
Distractor 2 & 2 & 2 & 0 & 0 & 2 & 2 \\
Noise 1 & 0 & 0 & 2 & 2 & 2 & 2 \\
Noise 2 & 0 & 0 & 2 & 2 & 2 & 2 \\
\hline
\end{tabular}

Inspection of the design used in Experiment 1 (Table 1) reveals that the four single-target displays occurred twice as often as the four redundant-target displays. Many previous studies have yielded faster responses to more frequent displays, both when displays vary in the frequency of a relevant stimulus (e.g., Miller \& Pachella, 1973) and when they vary in the frequency of an irrelevant attribute of a relevant stimulus (Miller, Schäffer, \& Hackley, 1991). In the detection task of Experiment 1, therefore, it was quite possible that response speed was also affected by display frequency, and that this effect may have contaminated the tests of the race-model inequality.

It is clear that the usual display-frequency effect-that is, faster responses to more frequent displays-would have worked against violations of the race-model inequality in Experiment 1 . The inequality is tested by comparing the RTs from single-target trials to the RTs from redundanttarget trials, and it is violated when redundant-target RTs are too fast. Because redundant-target displays were less frequent than single-target displays in Experiment 1, a frequency effect would have made it difficult for redundanttarget RTs to be fast enough (relative to single-target RTs) to produce violations. Thus, the effects of display frequency may have concealed evidence of coactivation. In Experiment 2, we tested for violations of the race-model inequality with display frequency equated across singleand redundant-target trials.

\section{Method}

The stimuli, equipment, and procedure from Experiment 1 were again employed. The only change from Experiment 1 occurred in the experimental design (compare Tables 1 and 3); under the design for Experiment 2, all displays appeared at the same frequency. In order to equate display frequency, while still avoiding biased contingencies, it was necessary to use two different types of distractor (i.e., there were two types of nontarget that could appear in the same display as a target). As in Experiment 1, there were two types of target and two types of noise. Twenty new subjects participated.

\section{Results}

The mean RTs from Experiment 2 appear in Table 4. The preliminary ANOVA again revealed a significant effect of practice $[F(1,19)=19.85, p<.001]$, but no interaction between practice and target condition $[F(2,38)=1.57, p>.10]$. Therefore, the data from Blocks 3-7 and Blocks 8-12 were combined for the tests for redundancy gains (but not in Table 4). Both types of redundant-target trial showed an RT advantage over single- target trials $[t(19)=9.76$ and 9.81 for same and different targets, respectively; both $p s<.001]$. Thirteen of the 20 subjects showed a positional preference, but the conservative tests also revealed significant redundancy gains $[t(19)=6.45$ and 7.15 for same and different targets; both $p \mathrm{~s}<.001]$. Experiment 2 exhibited no different-target advantage $[t(19)=0.43]$. After adjusting for the 11 subjects who showed a target preference (see Table 4), there was an insignificant advantage for the same-target condition $[t(19)=0.83]$.

The tests using the race-model inequality revealed significant evidence of coactivation (see Figure 2). For Blocks 3-7, the same-target data violated the race-model inequality from the 5 th to the 40 th percentiles (although none were significant), while the different-target data violated the equality at the 5th-30th percentiles, with those at the 5th-15th percentiles being significant (all $p s<.025$ or better). For Blocks 8-12, the same-target condition showed violations at the 5th-50th percentiles, with those at the 5th-25th percentiles being significant (all $p s<.05$ or better). There was an insignificant violation at the 5th percentile for the different-target condition for Blocks 8-12.

The subjects were again very accurate; the mean miss rate was $0.2 \%$ and the false-alarm rate was $2.5 \%$.

\section{Discussion}

The results from Experiment 2 are clearly inconsistent with both separate activations and the interactive race model, because violations of the race-model inequality were observed under an experimental design that included no biased contingencies. Thus, it appears that true coactivation is possible, at least in two-target tasks. When considered beside those from Experiment 1, these data also provide evidence that display frequency has an effect when subjects must search for two different targets. Experiment 1 included a display-frequency bias against redundanttarget trials and satisfied the race-model inequality. Experiment 2 did not include such a bias and showed sig-

Table 4

Mean Reaction Times (in Milliseconds), Experiment 2

\begin{tabular}{lcc}
\hline \multicolumn{1}{c}{ Condition } & Blocks $3-7$ & Blocks $8-12$ \\
\hline Single target, overall & 393.0 & 378.2 \\
Single target, conservative* & 386.6 & 369.9 \\
Same targets, overall & 360.0 & 349.3 \\
Same targets, conservative ${ }^{\dagger}$ & 357.0 & 341.9 \\
Different targets & 357.7 & 348.4 \\
\hline
\end{tabular}

*Corrected for favored positions. $\Varangle$ Corrected for preferred targets. 
Experiment 2
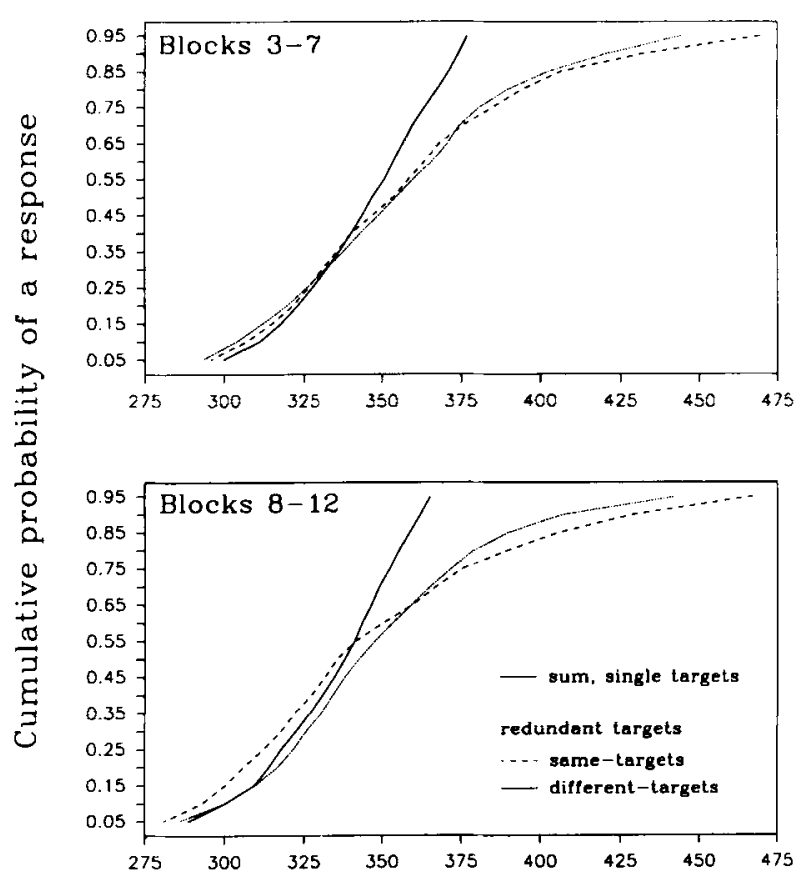

Time since stimulus onset (ms)

Figure 2. Cumulative distribution functions (CDFs) for Blocks 3-7 (upper panel) and Blocks 8-12 (lower panel), Experiment 2. (In each panel, the solid line represents the sum of the two single-target CDFs. The broken lines represent the redundant-target CDFs, with a dashed line for the same-target condition, and a dotted line for the differenttarget condition. The race-model inequality is often violated in the upper panel; there are violations for the same-target condition in the lower panel.)

nificant violations. In summary, these data suggest that when all known forms of bias are removed from the design of a two-target task (i.e., when there are no biased contingencies and when single- and redundant-target displays occur with equal frequencies), coactivation occurs on redundant-target trials.

At this point, two alternatives to our focus on display frequency need to be mentioned. First, one could focus instead on the frequencies at which each letter appeared at each location (cf. Miller, 1988; see also Reicher, Snyder, \& Richards, 1976). From this view, one would be interested in the marginal sums within Tables 1 and 2 , as opposed to the cell values. Such an analysis would yield a very similar interpretation of the present results: Under the design used for Experiment 2, all letters appeared at each location at the same frequency, whereas in Experiment 1, the distractor appeared twice as often at each location than did either of the targets. As in the display-frequency analysis that we favor, this would imply some advantage for single-target trials in Experiment 1 (which could conceal any evidence of coactivation), whereas Experiment 2 lacked such a bias.
Second, one could interpret the differences between Experiments 1 and 2 in terms of the number of different distractors. Because our two designs differed with respect to the number of distractors as well as relative frequency of single-target displays, it is logically possible that the former, rather than the latter, was responsible for violations of the race-model inequality observed in Experiment 2 . The individual and joint contributions of these two factors should be teased apart in future research, but for our main conclusion, the distinction is moot: Coactivation can be observed in a two-target task with unbiased contingencies, so the interactive race model cannot be generalized to apply to this situation.

Finally, we note that the results from Experiment 2 also imply that the different-target advantage is not as robust as might be inferred from Experiment 1 or the study by Grice and Reed (1992). Only the results from Blocks 8-12 of Experiment 1 showed an advantage for the differenttarget condition in the overall means, and the subjects in both of our experiments exhibited evidence of having a preferred target. Most importantly, in neither of our experiments did we observe a different-target advantage that could not be explained as being due to a target-preferences artifact.

\section{GENERAL DISCUSSION}

In the present study, we have explored several related issues concerning divided attention and the parallel processing of visual information. First, for the analysis of two-target data, we have adapted the notion of favored positions (Biederman \& Checkosky, 1970; Miller \& Lopes, 1988; Mullin et al., 1988) so that it can be applied to target preferences. This allowed us to test for one artifactual cause of the different-target advantage. In Experiment 1 , we replicated the different-target advantage in the overall means, but also found that it was due to target preferences; in Experiment 2, we found no differenttarget advantage. From these findings, we conclude that the different-target advantage is not a real effect, so it need not be explained and does not present a problem for the interactive race model.

Second, our results suggest that relative display frequency can have an important effect on divided-attention performance, although some of the effect may be carried by the number of different distractors. Under the design used for Experiment 1, each single-target display appeared twice as often as did each redundant-target display, and the results satisfied the race-model inequality. Under the design used for Experiment 2, single- and redundant-target displays appeared at the same frequency and the data violated the race-model inequality. This entire pattern of results suggests that including a displayfrequency bias in favor of single-target trials may conceal evidence of coactivation. More generally, these data are consistent with the view that display frequency has similar effects on target detection and on memory search (e.g., Miller \& Hardzinski, 1981). 
Most importantly, the present research has placed a constraint upon the interactive race model: namely, although the model accounts well for the performance of one-target tasks and provides an explanation for the effects of design contingencies, the model cannot account for all forms of divided attention. In particular, the model fails to explain the evidence of coactivation obtained in many twotarget tasks. Besides the present Experiment 2, Mordkoff and Yantis (1993, Experiments 1-3) have observed violations of the race-model inequality (under an unbiased experimental design) in a target-detection task that required attention to be divided between the separable dimensions of color and shape. From this it was concluded that redundant color and shape targets can coactivate (for a similar conclusion based on a different task, see Kubovy \& Cohen, 1991). Previously, Miller had provided evidence of coactivation in same-different tasks (1978) and bimodal target detection (1991), both of which involved at least two different stimulus dimensions and, therefore, more than one type of target.

The results from Experiment 2 may also provide some insight concerning the locus of coactivation. In general, two possible loci have been considered (see Fournier \& Eriksen, 1990; Grice \& Reed, 1992; Miller, 1982): one within perceptual processes, and one within postperceptual processes (i.e, at decision or motor-related stages). Perceptual coactivation involves the combining of information that concerns the presence of a specific target; postperceptual coactivation allows any activation in favor of a given response to be combined. Previous results have provided support for both of these loci. On the one hand, Miller (1991) observed larger redundancy gains when the two different targets were semantically related (e.g., a high-frequency tone and a light in the upper half of the display) than when the targets were incompatible (e.g., a high-frequency tone and a light in the lower half of the display). These data support a perceptual locus, because the results depended on the semantic relationship between the two targets (see also Fournier \& Eriksen, 1990). Giray and Ulrich (1993), on the other hand, observed more forceful responses to redundant targets, which suggests a postperceptual locus.

In the present Experiment 2, violations were observed for both the same- and different-target conditions during Blocks 3-7, but only the same-target condition showed violations during Blocks 8-12. In other words, the data from early blocks support a postperceptual locus, whereas the data from later blocks support a perceptual locus. Thus, our results suggest that practice with a task may alter the (active) locus of coactivation. When subjects have had little experience making responses to either or both of two different targets, coactivation may occur within postperceptual processes (and maybe within perceptual processes, as well). However, as subjects become more efficient at performing the task-possibly by learning to activate (go) responses based on weaker input to motorrelated stages-only a perceptual locus for coactivation remains detectable. One explanation of the practice effect, then, is that response activation becomes a "hair trigger," so that postperceptual coactivation becomes irrelevant. This would explain why only the same-target conditionwhich always would allow for perceptual coactivationviolates the race-model inequality during the late blocks of a testing session.

The multiple-locus coactivation model considered above is clearly incomplete, however, because it fails to explain why one-target tasks-which also include same-target trials-never show violations of the race-model inequality in the absence of biased contingencies. In general, the results from one-target tasks have consistently favored the interactive race model, whereas those from two-target tasks have often revealed evidence of coactivation. Taken at face value, this pattern of results appears somewhat counterintuitive; it would seem much more likely that inputs would be combined (i.e., allowed to coactivate) when there is only one type of target. Thus, a clear aim of future research is to identify the crucial distinctions between one- and two-target tasks that are responsible for this difference in the manner of parallel processing.

\section{REFERENCES}

Biederman, I., \& Checkosky, S. F. (1970). Processing redundant in formation. Journal of Experimental Psychology, 83, 486-490.

CoLonIUs, H. (1990). Possibly dependent probability summation of reaction time. Journal of Mathematical Psychology, 34, 253-275.

Diederich, A. (1992). Probability inequalities for testing separate activation models of divided attention. Perception \& Psychophysics, 52, 714-716.

Duncan, J. (1980). The locus of interference in the perception of simultaneous stimuli. Psychological Review, 87, 272-300.

ERIKSEN, C. W. (1988). A source of error in attempts to distinguish coactivation from separate activation in the perception of redundant targets. Perception \& Psychophysics, 44, 191-193.

Fournier, L. R., \& Eriksen, C. W. (1990). Coactivation in the perception of redundant targets. Journal of Experimental Psychology: Human Perception \& Performance, 16, 538-550.

GARNER, W. R. (1962). Uncertainty and structure as psychological concepts. NeW York: Wiley.

GIRAY, M., \& ULRICH, R. (1993). Motor coactivation revealed by response force in divided and focused attention. Manuscript submitted for publication.

Grice, G. R., \& CANHAM, L. (1990). Redundancy phenomena are affected by response requirements. Perception \& Psychophysics, 48, 209-213.

Grice, G. R., Canham, L., \& Boroughs, J. M. (1984). Combination rule for redundant information in reaction time tasks with divided attention. Perception \& Psychophysics, 35, 451-463.

Grice, G. R., \& ReED, J. M. (1992). What makes targets redundant? Perception \& Psychophysics, 51, 437-442.

Kubovy, M., \& COHEN, D. (1991, November). Are texture segregation by form and color independent of each other? Paper presented at the meeting of the Psychonomic Society, San Francisco, CA.

MILLER, J. (1978). Multidimensional same-different judgments: Evidence against independent comparisons of dimensions. Joumal of Experimental Psychology: Human Perception \& Performance, 4, 411-422.

MILLER, J. (1982). Divided attention: Evidence for coactivation with redundant signals. Cognitive Psychology, 14, 247-279.

MiLleR, J. (1988). Components of the location probability effect in visua search tasks. Journal of Experimental Psychology: Human Perception \& Performance, 14, 453-471. 
Miller, J. (1991). Channel interaction and the redundant-targets effect in bimodal divided attention. Journal of Experimental Psychology: Human Perception \& Performance, 17, 160-169.

Miller, J., \& Hardzinski, M. (1981). Case specificity of the stimulus probability effect. Memory \& Cognition, 9, 205-216.

Miller, J., \& LOPES, A. (1988). Testing race models by estimating the smaller of two true mean or true median reaction times: An analysis of estimation bias. Perception \& Psychophysics, 44, 513-524.

Miller, J., \& LoPES, A. (1991). Bias produced by fast guessing in distribution-based tests of race models. Perception \& Psychophysics, 50, 584-590.

Miller, J., \& Pachella, R. (1973). Locus of the stimulus probability effect. Journal of Experimental Psychology, 101, 227-231.

Miller, J., SChÄFfER, R., \& HACKLEY, S. A. (1991). Effects of preliminary information in a go versus no-go task. Acta Psychologica, 76, 241-292.

MoRdKoff, J. T. (1992). Testing race models of the redundant-signals effect: A warning concerning the combination-rule regression analysis. Perception \& Psychophysics, 52, 589-594.

MORDKOFF, J. T., \& Y ANTIS, S. (1991). An interactive race model of divided attention. Journal of Experimental Psychology: Human Perception \& Performance, 17, 520-538.

MordKoff, J. T., \& Y ANTIS, S. (1993). Dividing attention between color and shape: Evidence of coactivation. Perception \& Psychophysics, 53, 357-366.

Mullin, P. A., Egeth, H. E., \& Mordoroff, J. T. (1988). Redundanttarget detection and processing capacity: The problem of positional preferences. Perception \& Psychophysics, 43, 607-610.

RAAB, D. (1962). Statistical facilitation of simple reaction time. Transactions of the New York Academy of Sciences, 43, 574-590.

Reicher, G. M., SNYDER, C. R. R., \& Richards, J. T. (1976). Familiarity of background characters in visual scanning. Journal of Experimental Psychology: Human Perception \& Performance, 2, 522-530.

SCHWARZ, W. (1989). A new model to explain the redundant-signals effect. Perception \& Psychophysics, 46, 498-500.

SHAW, M. L. (1982). Attending to multiple sources of information: I. The integration of information in decision making. Cognitive Psychology, 14, 353-409.

TownSEnd, J. T., Nozawa, G. (1992). On the spatio-temporal properties of elementary perception: An investigation of parallel, serial, and coactive theories (Rep. No. 65). Bloomington, IN: Indiana University, Cognitive Science Program.

ULRICH, R., \& GIRAY, M. (1986). Separate-activation models with variable base times: Testability and checking of cross-channel dependency. Perception \& Psychophysics, 39, 248-254.

van der Heijden, A. H. C. (1975). Some evidence for a limited capacity parallel self-terminating process in simple visual search tasks. Acta Psychologica, 39, 21-41. van der Heijden, A. H. C., Schreuder, R., Maris, L., \& NeerINCX, M. (1984). Some evidence for correlated separate activation in a simple letter-detection task. Perception \& Psychophysics, 36, 577-585.

\section{NOTES}

1. Serial models have also been considered, but these models are ruled out by studies in which the number of display items, as well as the number of targets, has been varied. For example, van der Heijden (1975) compared the reaction times (RTs) from trials with one target alone in the display with the RTs from trials with two or three targets. Serial models predict no redundancy gain for this situation, because the first letter to be processed would be a target in every case, yet a redundancy gain was observed (see also, Grice \& Canham, 1990; Mordkoff \& Yantis, 1991; van der Heijden et al., 1984).

2. Target preferences can also produce an artifactual different-target advantage under an interactive race model or a coactivation model. The interactive race model differs from the independent race model in allowing the speed of the racers to be influenced by interstimulus contingencies, but Equation 4 still holds because the RT on a redundant-target trial is determined by the winner of a race. The variety of mathematical forms available for coactivation models prevents us from proving that an artifactual different-target advantage arises under every member of this class, but simulations reveal that it does occur within at least some plausible examples (e.g., Mordkoff, 1992; Schwarz, 1989).

3. We originally analyzed the present data in a manner similar to that of Grice and Reed (1992), by combining the CDFs from Blocks 3-7 and Blocks 8-12 before conducting tests of the race-model inequality. More recent results from experiments involving two different targets, in which stable results were only observed after some practice, led us to the present analysis.

4. It should be noted that our conservative test for a different-target advantage only corrects for a fixed preferred target (cf. fixed favored positions; Miller \& Lopes, 1988). Our test does not correct for any artifacts due to a preference that changes between trials, nor is such a test currently available (see Mullin et al., 1988). In addition, our test fails to counteract differences in single-target distributions other than those that would alter mean RT (cf. Equation 4). Thus, there may still be a processing advantage for same-preferred-target trials over different-target trials, despite our inability to observe it. (We thank a reviewer for raising this issue.)

(Manuscript received July 31, 1992; revision accepted for publication October 23, 1992.) 